\title{
Does the evidence support population-wide screening for type 2 diabetes? No
}

\author{
Jonathan E. Shaw ${ }^{1}$ (D) \\ Received: 21 June 2017 / Accepted: 28 June 2017 /Published online: 23 August 2017 \\ (C) Springer-Verlag GmbH Germany 2017
}

\begin{abstract}
Large-scale, centrally-coordinated screening for undiagnosed type 2 diabetes is an attractive option to reduce the mortality and morbidity resulting from inadequately controlled diabetes. However, there is limited research examining the direct consequences of such screening programmes on outcomes such as cardiovascular disease and death. Two papers published in this edition of Diabetologia (DOIs: 10.1007/s00125-0174323-2 and 10.1007/s00125-017-4299-y) examine data from one of the very few trials conducted in this area. Overall, there was little benefit that could be directly related to the screening programme. In part, this was due to the high levels of opportunistic screening in the control group. Thus, when there are high levels of opportunistic screening for type 2 diabetes, there remains no clear evidence of benefit of centrally-coordinated screening programmes that approach individuals outside usual healthcare settings.
\end{abstract}

Keywords Cardiovascular disease $\cdot$ Mortality $\cdot$ Screening · Type 2 diabetes
Abbreviations
ADDITION Anglo-Danish-Dutch Study of Intensive
Treatment in People with Screen-Detected
Diabetes in Primary Care
CVD Cardiovascular disease

Jonathan E. Shaw

jonathan.shaw@bakeridi.edu.au

1 Baker Heart and Diabetes Institute, Level 4, 99 Commercial Road, Melbourne, VIC 3004, Australia

\section{Background to screening}

Screening for disease implies classifying asymptomatic individuals according to their probability of disease, identifying those who satisfy diagnostic criteria, and intervening in order to prevent or delay morbidity and death. It can occur under two broad circumstances: (1) opportunistic screening examines people who are presenting to health services for some other reason; (2) community screening involves approaching the general population, through direct outreach or in nonhealthcare settings. Examples of community screening include breast and colon cancer screening programmes in middle-aged adults. It is this latter type of screening that we focus on here.

The appeal of screening lies in the early identification of a disease process, before symptoms appear, and the timely treatment to prevent, slow or reverse the disease. Furthermore, it is often perceived as cost-saving, as screening and early intervention are typically less expensive than treatment of severe disease, at least for a single individual. However, despite the appeal, there are many potential challenges. Screening and diagnostic tests can have uncertainties and their own adverse effects, and may lead to a series of unfruitful and costly further investigations. Even if left untreated, a proportion of those identified as having the disease will never actually develop symptoms or disease complications, as the disease may run a very benign course. Alternatively, a more aggressive disease may supervene before screening has time to benefit the primary disease of concern. To establish a screening programme, it needs to be clear that interventions are both available and effective, that there is a prolonged pre-clinical phase, and that the benefits and harms of the interventions have been demonstrated in an asymptomatic population. Finally, since screening programmes typically test and intervene in far more people than will ever develop significant disease consequences, 
the population costs for even cheap testing approaches can far outweigh cost savings.

On the face of it, type 2 diabetes satisfies many of the criteria established for screening programmes [1]; it has a long asymptomatic period (during which serious complications can develop), there are screening tools with reasonable predictive performance $[2,3]$, the diagnostic tests are relatively simple and cheap, and interventions for glucose, blood pressure and lipids are effective [4-6]. However, unlike screening for other conditions, such as breast and colon cancer [7], screening for type 2 diabetes has not been subjected to formal clinical trials. This is partly because of the challenges associated with designing appropriate studies. Unlike common cancers, the relevant outcomes of diabetes within a screened population are either too rare and take too long to occur (e.g. kidney failure), or are not possible to attribute to diabetes (e.g. myocardial infarction), especially in a setting where diabetes itself may have remained undiagnosed.

\section{Recent lessons from the Anglo-Danish-Dutch Study of Intensive Treatment in People with Screen-Detected Diabetes in Primary Care (ADDITION) trial}

The Anglo-Danish-Dutch Study of Intensive Treatment in People with Screen-Detected Diabetes in Primary Care (ADDITION) is the only trial that has attempted to establish the effects of diabetes screening [8]. The ADDITION research team undertook a large diabetes screening programme using general practices in three European countries and then, at the practice level, randomised individuals with screen-detected diabetes to intensive or conservative multifactorial therapy. Thus, this was not a true study of the population-level impact of screening, but instead tested intensive management of blood glucose levels and cardiovascular risk, which in this setting was the immediate consequence of screening. Unfortunately, the main trial results were rather inconclusive, showing a non-significant reduction in macrovascular and microvascular complications [8]. A series of subsequent analyses has attempted to provide more clarity. For example, Herman et al modelled the trial data and suggested a mortality benefit after 5 years as a result of diagnosing and treating diabetes 3 years earlier through screening [9].

In this edition of Diabetologia, two further analyses of the Danish component of ADDITION are presented. These two analyses attempt to look more specifically at the effects of screening, by comparing the populations in general practices taking part in the ADDITION screening programme with populations from other practices outside of the ADDITION trial. In the first, Simmons et al took a population perspective of the ADDITION trial [10]; the analysis compared mortality and non-fatal cardiovascular disease (CVD) event rates in individuals aged 40-69 years in the whole population of the 181 general practices participating in ADDITION-Denmark with the rest of the Danish population of the same age (using data from Danish national registers). Over a median follow-up period of 6.2 years, the two populations had almost identical mortality and CVD event rates, indicating no effect of diabetes screening at the population level. This is disappointing, but perhaps not surprising, as individuals with diabetes that are identified through screening comprise only a small proportion of the total number of people who will have a CVD event or will die in the general population. Since most people with these events do not have diabetes, the capacity to influence mortality and CVD event rates at the population level would be very small, even if the early identification of diabetes through screening genuinely improved outcomes for screenpositive individuals.

In the second paper [11], Simmons and colleagues compared the mortality and non-fatal CVD outcomes of those newly diagnosed with diabetes in the practices included in the ADDITION screening programme (including individuals identified through the screening programme as well as those identified through standard clinical procedures) with those who were diagnosed through usual processes in the rest of Denmark. The main finding is that people who were identified with diabetes in the screening practices had $21 \%$ and $16 \%$ lower mortality and CVD rates, respectively, compared with people diagnosed with diabetes in the rest of the country. This is an interesting finding but the analysis was non-randomised, comparing those practices volunteering to take part in ADDITION with all other practices. Some reassurance that this finding is not driven by an inherently lower CVD risk in the ADDITION population compared with the rest of the Danish population is actually provided by the previous paper by Simmons et al (discussed above) [10], which showed almost identical outcome rates in the two general populations. But can this apparent benefit be attributed to the screening programme? The uptake of the screening programme was fairly modest and, in fact, only $10 \%$ of all people newly diagnosed with diabetes in the ADDITION practices were actually identified through the screening programme; the remaining $90 \%$ were diagnosed in standard clinical settings. It seems highly unlikely that aggressive management of these screenpositive individuals could have led to the lower event rates seen in the whole group. Thus, perhaps there was better management of diabetes in the ADDITION practices than elsewhere, but there is no clear evidence that early identification through screening was beneficial. Furthermore, those individuals identified as having diabetes through screening are typically earlier in the disease process than are those who are clinically diagnosed, and would therefore be expected to have lower event rates over a given period of time, even if no effective treatment is provided. A similar phenomenon likely also explains some, or perhaps all, of the survival benefit 
associated with diabetes screening in a report of a Swedish screening programme [12]. This lead-time bias is a wellrecognised problem in the evaluation of screening programmes and is the main reason for preferring whole-ofpopulation analyses over those focussing on the diagnosed cases.

\section{Implications for screening for type 2 diabetes}

In sum, these new analyses do not change the balance of evidence to warrant population-wide screening for diabetes. However, it is important to recognise that ADDITION tested a community screening programme, not opportunistic screening. It compared a centrally-coordinated programme of screening with usual care, which itself likely included a fair amount of opportunistic screening. Indeed, studies from highincome countries have demonstrated an increase over time in laboratory testing for diabetes and that this occurred in the absence of any formal screening programmes or recommendations [13]. Thus, in both study arms, asymptomatic individuals with risk factors for type 2 diabetes were being tested for the presence of diabetes. In one arm, this was part of usual care, in the other this usual care was supplemented with a screening programme. Furthermore, if there is good management of cardiovascular risk factors, even people with undiagnosed diabetes may have a fairly low cardiovascular event rate.

The results might have been different if the uptake of screening had been higher than the $18 \%$ achieved in ADDITION. Experience from screening in other conditions, like colon cancer screening, indicate that considerably higher uptake is achievable in some screening programmes [14]. ADDITION used only a single mailed invitation, with no reminders for non-responders. The results might also have differed if clinical diagnostic rates were lower. When clinical diagnostic rates are high because of already active opportunistic screening processes, there is little opportunity for a centrally-coordinated screening programme to add very much. Another important influence on the success of screening is the effectiveness (and cost-effectiveness) of available treatments for those found to have diabetes. Currently-available therapies for type 2 diabetes are, at best, of modest efficacy. However, if the impressive effects of sodium-glucose cotransporter 2 (SGLT2) inhibitors on cardiovascular outcomes among people with diabetes who are at very high cardiovascular risk [15, 16] were also shown to apply to those at lower cardiovascular risk, this might influence the screening argument.

It is important to note what has not been shown by ADDITION. First, it has not been shown that opportunistic screening is ineffective, as no trials have actually tested this. Second, it has not shown that screening programmes are ineffective in settings of low clinical diagnosis rates. This may be relevant for lower- and middle-income countries, where clinical diagnosis may be significantly delayed and the complication rates are high. However, even in these settings, careful thought needs to be given to deciding whether scarce resources are spent on identifying early disease or on treating the complications of established clinical disease. It is unlikely that a screening trial could be run in such settings, as the very circumstances that lead to low levels of diagnosis often go hand in hand with a lack of the death and cardiovascular event registrations that are needed for these trials. Modelling approaches should be considered to address this question.

Although the evidence for community screening is not strong, it is necessary to recognise that the success of screening programmes is very sensitive to the circumstances in which they are set. The potential reach and acceptability of the screening tests, the background level of screening and diagnosis through clinical channels, and the efficacy of available interventions all have profound effects on the performance of a screening programme. Thus, the question of community screening for diabetes needs to be reassessed according to local settings and over time, and not as a one-off decision based on a single study.

\section{Summary}

The appropriate conclusion from the currently-available evidence is that community screening programmes, such as those that have been established for colon and breast cancer, cannot be justified for type 2 diabetes in countries where opportunistic diabetes screening is functioning well, and management of cardiovascular risk factors is good. The large amounts of public money required for such screening programmes would be better spent on treating those with clinically diagnosed disease. The question remains open in less-developed healthcare systems, where the prevalence of undiagnosed diabetes is high and screening offers an opportunity to initiate effective care, but possibly at the expense of resources that are sorely needed for people with established disease.

Acknowledgements E. Gregg (Centers for Disease Control and Prevention, Atlanta, GA, USA) is acknowledged for providing comments and advice on this commentary.

Funding JS is supported by a National Health and Medical Research Council Fellowship (APP1079438).

Duality of interest JS has received honoraria for advisory boards and lectures from AstraZeneca, BGP Products, Boehringer Ingelheim, Eli Lilly, Janssen, Merck Sharp \& Dohme, Novartis, Novo Nordisk, and Sanofi. 
Contribution statement The author was the sole contributor to this paper.

\section{References}

1. Wilson JMG, Jungner G (1968) Principles and practice of screening for disease. World Health Organization, Geneva

2. Tanamas SK, Magliano DJ, Balkau B et al (2015) The performance of diabetes risk prediction models in new populations: the role of ethnicity of the development cohort. Acta Diabetol 52:91-101

3. Noble D, Mathur R, Dent T, Meads C, Greenhalgh T (2011) Risk models and scores for type 2 diabetes: systematic review. BMJ 343: d7163

4. Holman RR, Paul SK, Bethel MA, Matthews DR, Neil HA (2008) 10-year follow-up of intensive glucose control in type 2 diabetes. N Engl J Med 359:1577-1589

5. Emdin CA, Rahimi K, Neal B, Callender T, Perkovic V, Patel A (2015) Blood pressure lowering in type 2 diabetes: a systematic review and meta-analysis. JAMA 313:603-615

6. Cholesterol Treatment Trialists' (CTT) Collaborators (2008) Efficacy of cholesterol-lowering therapy in 18,686 people with diabetes in 14 randomised trials of statins: a meta-analysis. Lancet 371:117-125

7. Lin JS, Piper MA, Perdue LA et al (2016) Screening for colorectal cancer: a systematic review for the U.S. Preventive Services Task Force. Agency for Healthcare Research and Quality (US), Rockville (MD)

8. Griffin SJ, Borch-Johnsen K, Davies MJ et al (2011) Effect of early intensive multifactorial therapy on 5-year cardiovascular outcomes in individuals with type 2 diabetes detected by screening
(ADDITION-Europe): a cluster-randomised trial. Lancet 378: 156-167

9. Herman WH, Ye W, Griffin SJ et al (2015) Early detection and treatment of type 2 diabetes reduce cardiovascular morbidity and mortality: a simulation of the results of the Anglo-Danish-Dutch Study of intensive treatment in people with screen-detected diabetes in primary care (ADDITION-Europe). Diabetes Care 38:14491455

10. Simmons RK, Griffin SJ, Witte DR, Borch-Johnsen K, Lauritzen T, Sandbæk A (2017) Effect of population screening for type 2 diabetes and cardiovascular risk factors on mortality rate and cardiovascular events: a controlled trial among 1,912,392 Danish adults. Diabetologia doi:10.1007/s00125-017-4323-2

11. Simmons RK, Griffin SJ, Lauritzen T, Sandbæk A (2017) Effect of screening for type 2 diabetes on risk of cardiovascular disease and mortality: a controlled trial among 139,075 individuals diagnosed with diabetes in Denmark between 2001 and 2009. Diabetologia doi:10.1007/s00125-017-4299-y

12. Feldman AL, Griffin SJ, Fhärm E et al (2017) Screening for type 2 diabetes: do screen-detected cases fare better? Diabetologia doi:10.1007/s00125-017-4402-4

13. Wilson SE, Lipscombe LJ, Rosella LC, Manuel DG (2009) Trends in laboratory testing for diabetes in Ontario, Canada 1995-2005: a population-based study. BMC Health Serv Res 9:41

14. Centers for Disease Control and Prevention (CDC) (2013) Vital signs: colorectal cancer screening test use - United States, 2012. MMWR 62:881-888

15. Zinman B, Wanner C, Lachin JM et al (2015) Empagliflozin, cardiovascular outcomes, and mortality in type 2 diabetes. $\mathrm{N}$ Engl $\mathrm{J}$ Med 373:2117-2128

16. Neal B, Perkovic V, Mahaffey KW et al (2017) Canagliflozin and cardiovascular and renal events in type 2 diabetes. N Engl J Med doi:10.1056/NEJMoa1611925 